\title{
Branched chain amino acids and metabolic regulation
}

\author{
WANG ChunXia \& GUO FeiFan* \\ Key Laboratory of Nutrition and Metabolism, Institute for Nutritional Sciences, Shanghai Institutes for Biological Sciences, Chinese Academy of \\ Sciences, Shanghai 200031, China
}

Received September 25, 2012; accepted November 30, 2012; published online January 21, 2013

\begin{abstract}
Amino acids are fundamental nutrients required for protein synthesis. The branched chain amino acids (BCAAs) leucine, isoleucine, and valine are the most abundant of the essential amino acids. BCAAs have recently been recognized as having functions in processes other than simple nutrition. For example, metabolic diseases are characterized by higher levels of circulating BCAAs. Moreover, supplementation with or deficiency in BCAAs is closely related to the regulation of metabolic homeostasis. Indeed, leucine deprivation induces increased lipolysis and thermogenesis, which result in fat loss, as well as suppressed lipogenesis and enhanced insulin sensitivity in the liver. Accumulating evidence has indicated that several amino acid sensors, including GCN2, ATF4, mTOR, and AMPK, play pivotal roles in the regulation of lipid metabolism, glucose metabolism, and energy homeostasis. Furthermore, the hypothalamus is critical for sensing amino acid levels and mediates the metabolic adaptation of the body upon limitation of essential amino acids (EAAs) through regulating expression of the S6K1, MC4R, and CRH. In this review, we highlight recent studies investigating the cellular mechanisms linking amino acids, amino acid sensors, metabolic regulation, and metabolic diseases. Amino acid sensing and metabolic regulation have become research hotspots in the metabolic field.
\end{abstract}

branched chain amino acids, amino acid sensor, lipid metabolism, glucose metabolism, energy homeostasis, obesity, diabetes

Citation: $\quad$ Wang C X, Guo F F. Branched chain amino acids and metabolic regulation. Chin Sci Bull, 2013, 58: 1228-1235, doi: 10.1007/s11434-013-5681-x

Metabolic diseases, including obesity, fatty liver, and diabetes, have become a major public health problem, and their prevalence has increased dramatically over the last few decades among all ages worldwide. The obesity epidemic has been recognized by the World Health Organization (WHO) as one of the top 10 global health problems. Obesity has reached epidemic proportions in many countries around the world and is strongly linked to a number of chronic diseases, including diabetes, hypertension, and cardiovascular disease. Worldwide, more than 1 billion adults are overweight and over 300 million are obese [1]. Data on the prevalence of diabetes by age and sex from all 191 WHO member states revealed that the estimated prevalence of diabetes for all age groups worldwide was $2.8 \%$ in 2000 and is expected to increase to $4.4 \%$ by 2030 . The total number of people with diabetes is projected to rise from 171 million in 2000 to 366 million in 2030 [2].

*Corresponding author (email: ffguo@sibs.ac.cn)
In China, the rapid transformation from a rural agrarian society to an industrial society with increased wealth has impacted the health of the entire population, especially evident by the increasing high prevalence of metabolic diseases. Obesity, especially abdominal obesity, contributes to many metabolic disorders, including metabolic syndrome (MetS), type 2 diabetes (T2DM), and cardiovascular diseases (CVDs). In China, more than one-third of adults are overweight or obese and 10\%-20\% of all adults are affected by MetS [3]. The rapid growth of the overweight/obese population in China will undoubtedly raise the worldwide prevalence of chronic disease. Indeed, diabetes has reached epidemic proportions in the general adult population in China. Overall, 92.4 million adults 20 years of age or older $(9.7 \%$ of the adult population) have diabetes, and $60.7 \%$ of these cases remain undiagnosed [4]. Fatty liver (steatosis) is highly prevalent in China and is often linked to obesity. The community prevalence of non-alcoholic fatty liver disease (NAFLD) is approximately $15 \%$, and the prevalence of 
NAFLD has doubled in the past decade [5]. Metabolic diseases, including obesity, fatty liver, and diabetes have become a major public health problem in China, and strategies aimed at the prevention and treatment of such conditions are needed. Moreover, the relative contribution of nutritionrelated chronic diseases to the total disease burden of the society and health care costs has risen continuously over the last few decades. Although a number of pharmacological approaches have been investigated in recent years, few safe, therapeutically effective products have been developed. Thus, there is an urgent need to better exploit the potential of disease prevention via dietary changes.

Amino acids are fundamental nutrients required for protein synthesis. Twenty amino acids, termed proteinogenic or standard amino acids, are naturally incorporated into polypeptides. Among them, the branched chain amino acids (BCAAs) leucine, isoleucine, and valine are the most abundant of the essential amino acids (EAAs) and cannot be created from other compounds by the human body; therefore, they must be ingested in the form of food. Amino acids are important in nutrition and are commonly used in nutritional supplements, fertilizers, food technology, and in the industry. Recently, accumulating evidence has indicated that BCAAs have essential roles in the physiological regulation of many processes besides simple nutrition. In this review, we will provide an overview on the function of BCAAs in the regulation of metabolic processes in the body, including lipid metabolism, glucose metabolism, and energy homeostasis, and the effects of these BCAAs on the development of metabolic disease.

\section{Amino acids and obesity}

Obesity is a condition associated with the accumulation of excessive body fat resulting from chronic imbalance of abundant energy intake and low energy expenditure [6]. Various strategies have been proposed to treat obesity by promoting fat mobilization, increasing energy expenditure, and/or reducing food intake [1,7]. Accumulating evidence has shown that diets with higher protein and reduced carbohydrates are beneficial for weight loss and increase loss of body fat [8-10]. Because leucine can interact with the insulin-signaling pathway and appears to regulate the oxidative use of glucose by skeletal muscle through stimulation of glucose recycling via the glucose-alanine cycle, the key to understanding the relationship between dietary protein and carbohydrates is the relationship between the intake of leucine and glucose [11].

It has long been recognized that several amino acids, including BCAAs, are elevated in the blood of obese subjects relative to healthy individuals [12]. In 2009, to gain a broader understanding of the metabolic and physiological differences between obese and lean subjects, Newgard et al. [13] applied comprehensive metabolic profiling tools to compare the metabolic profiles of obese versus lean humans; their data revealed that increased catabolism of BCAAs correlated with obesity. This was the first direct report demonstrating the relationship between BCAAs and metabolic signatures in the serum of obese individuals. Zhang et al. [14] demonstrated that doubling the intake of dietary leucine without increasing food intake decreased adiposity by $25 \%(P<0.01)$ in mice fed a high-fat diet $(\mathrm{HFD})$; this change was a result of increased resting energy expenditure associated with increased expression of uncoupling protein 3 in brown and white adipose tissue (BAT and WAT, respectively) and in skeletal muscle [14]. The effect of increasing dietary leucine, however, is still controversial. Additional studies have shown that dietary supplementation of leucine has no effect on lipid metabolism [15]. In contrast, Guo et al. [16] found that mice maintained on a leucine-deficient diet for $7 \mathrm{~d}$ experienced a dramatic reduction in abdominal fat mass and differential lipid metabolism in the liver, which was the first report of the effects of leucine deprivation on lipid metabolism and was significant enough to be highlighted in Nature Medicine.

Food intake determines energy intake, which plays a key role in the development of obesity. Recently, increased hypothalamic availability of the BCAA leucine was shown to act as a potent signal for reducing food intake [17]. Additionally, supplementation of an HFD with BCAAs (HF/BCAA) reduces food intake and body weight [13]. In contrast, our research work indicated that leucine deprivation for $7 \mathrm{~d}$ resulted in a $15 \%$ reduction in food intake and body weight and an approximately 50\% reduction in abdominal fat compared with those in mice maintained on a control diet [18]. Furthermore, $1.1 \mu \mathrm{g}$ leucine in $1.0 \mu \mathrm{L}$ PBS administered by intravenous injection once a day for $7 \mathrm{~d}$ did not influence food intake, but modestly blocked body weight reduction in mice maintained on a leucine-deficient diet [19]. In addition to many identified hypothalamic pathways controlling food intake [20], these results suggested that leucine deprivation induces decreased food intake and that leucine also is a key hypothalamic regulator of food intake in mice.

Enhanced lipid mobilization is an effective method for reducing fat. In contrast with work demonstrating that the addition of leucine improves insulin sensitivity and the downregulation of hepatic glucose-6-phosphatase [14], Guo's research further showed that leucine deprivation accelerates triglyceride lipolysis and $\beta$-oxidation gene expression in WAT. In a study that was the first to demonstrate the relationship between leucine and metabolism, researchers showed that the levels of phosphorylated HSL (p-HSL) and mRNAs encoding the transcription factor peroxisome proliferator-activated receptor $\alpha$ and its target genes carnitine palmitoyltransferase $1(\mathrm{Cptl})$ and fatty acyl-CoA oxidase $(A c o)$ significantly increased in the WAT of leucine-deprived mice [18]. Moreover, lipogenic genes, including acetyl CoA carboxylase 1 (Accl), fatty acid syn- 
thase (Fas), and stearoyl CoA desaturase $(S c d)-1$, are all suppressed in the WAT of mice fed a leucine-deficient diet for $7 \mathrm{~d}$ [18].

BAT plays a pivotal role in the prevention or treatment of obesity, and the identification of BAT in adult humans was reported in 2009 [21]. Interestingly, elimination of leucine from the diet can significantly increase energy expenditure, which mainly contributes by increasing thermogenesis due to upregulation of UCP1 expression in BAT. Additionally, leucine deprivation activates the expression of $A d r b 3$, the main $\beta$-adrenoceptor isoform in BAT and WAT, which suggests that the sympathetic nervous system is involved in the regulation of leucine deprivation-induced fat loss [18]. Although the mechanisms underlying the leucine deprivation-induced regulation of thermogenesis are largely unclear, models incorporating use of leucine-deficient diets give new insight into the dietary prevention of obesity.

Guo's group also demonstrated that, in addition to leucine, isoleucine- or valine-deficient diets could also induce significant fat loss due to enhanced lipid mobilization and increased energy expenditure [22]. Subsequently, a question arises regarding how BCAA deprivation induces fat loss with increased energy expenditure. In a previous report, knockout of the BCATm gene, encoding the enzyme catalyzing the first step in peripheral BCAA metabolism, resulted in decreased adiposity and body weight along with increased energy expenditure and protection from dietinduced obesity in mice. Increased energy expenditure was strongly associated with food consumption and an active futile cycle of increased protein degradation and synthesis [23]. These results suggest that BCAA metabolism is closely related to protein metabolism and the regulation of energy homeostasis.

\section{Amino acids and diabetes}

Several amino acids, including BCAAs, have long been known to be elevated in the blood of patients with insulin-resistance or type 2 diabetes relative to healthy control individuals [12]. In 2009, Newgard et al. [13] applied comprehensive metabolic profiling tools to reveal that increased catabolism of BCAA was correlated with insulin resistance. Recently, Wang et al. [24] reported that isoleucine, leucine, valine, tyrosine, and phenylalanine had highly significant associations with future diabetes. This evidence suggests that amino acids, especially BCAAs, are potentially important biomarkers for the diagnosis of diabetes and prediabetes.

However, this is inconsistent with the fact that supplementation with or deletion of amino acids affects insulin sensitivity. Interestingly, nutritional supplementation with a special mixture of oral amino acids also significantly improves insulin sensitivity in elderly subjects with type 2 diabetes [4]. In addition, supplementation with BCAAs has the potential to improve glucose metabolism in patients with liver cirrhosis [25]. BCAAs, especially leucine, are closely related to glucose metabolism and insulin sensitivity both in vivo and in vitro. Zhang and colleagues [14] recently demonstrated that increased oral intake of leucine improves whole-body insulin sensitivity, decreases plasma concentrations of glucagon and glucogenic amino acids, and downregulates hepatic glucose-6-phosphatase in mice maintained on an HFD. However, the effect of increasing dietary leucine remains controversial. Some reports have shown that increased serum levels of leucine have no effect on insulin sensitivity [15], while others have shown that increase serum leucine promotes insulin resistance in humans and animal models of obesity $[13,26]$. In contrast, dietary leucine deprivation decreases serum insulin levels 3 -fold in mice with normal glucose levels, suggesting that increased insulin sensitivity may occur in response to leucine deprivation [16]. Further work has shown that leucine deprivation improves hepatic insulin sensitivity by sequentially activating general control nonderepressible 2 (GCN2) and decreasing mammalian target of rapamycin (mTOR) signaling, as well as activating AMP-activated protein kinase (AMPK) in the context of leucine deprivation [27]. Moreover, leucine deprivation also improves insulin sensitivity in nutritionally or genetically induced insulin resistance models, which provides hope to patients with diabetes.

Through analyzing amino acid levels in the serum of leucine-deprived mice, Guo's group found that these mice displayed decreased leucine, but increased isoleucine and valine in the serum. Because the levels of all 3 of these BCAAs, as well as other amino acids, are elevated in insulin-resistant conditions in vivo $[13,26]$ and in vitro [28], the low level of leucine may play a key role in promoting enhanced insulin sensitivity in mice fed a leucine-deficient diet. Unpublished data from our laboratory suggest that isoleucine or valine deprivation also improves insulin sensitivity via a different mechanism.

Although leucine is recognized as an important nutrient regulator involved in metabolic processes, the mechanisms through which leucine signaling or BCAA metabolism is related to the regulation of insulin sensitivity and glucose metabolism have not yet been elucidated. Recently, Higuchi et al. [29] indicated that BCAA administration enhanced the mRNA expression of $L-G k$, Srebp-1c, Lxro, and Chrebp and suppressed the expression of G6pase mRNA in the rat liver without affecting glycogen synthase expression or serum glucose concentrations. These results suggest that BCAA metabolism is closely related to insulin sensitivity and glucose metabolism. However, it is currently unclear how amino acid-stimulated signal transduction pathways affect insulin sensitivity. One hypothesis is that the amino acid transporter plays a critical role in this process, which has been illustrated in a previous study [30]. Another possibility is that enzymes catalyzing steps in peripheral BCAA metabolism mediate amino acid-regulated insulin sensitivity 
or glucose homeostasis. For example, it was previously reported that knockout of the BCATm gene in mice resulted in marked improvement in glucose and insulin tolerance [23].

\section{Amino acid sensors and metabolic diseases}

Amino acid sensing is a critical process that enables cells to respond properly to changes in amino acids levels in order to maintain metabolic homeostasis. Amino acid sensors or transporters play key roles in these processes. Great progress has been made in studying the mechanisms underlying amino acid sensing in unicellular organisms, i.e., yeast and Escherichia coli. However, it is still unclear how the body senses amino acids levels to control metabolic homeostasis, including lipid metabolism, glucose metabolism, and energy homeostasis.

Currently, amino acid sensing and metabolic regulation have become a research hotspot in the metabolic field. Besides focusing on the effects of EAAs on metabolic regulation, Guo's laboratory is also interested in exploring novel functions of certain proteins implicated in amino acid regulatory pathways, including GCN2, ATF4, mTOR, AMPK, etc (Figure 1).

\subsection{GCN2}

GCN2 is recognized as a sensor for amino acid deprivation. As a kinase, GCN2 is activated by uncharged tRNAs in response to the deprivation of EAAs in yeast and mammals [31]. Activated GCN2 phosphorylates eukaryotic initiation factor $2-\alpha(\mathrm{eIF} 2 \alpha)$, resulting in the repression of general protein synthesis and increased translation of proteins related to amino acid biosynthesis and transport [32]. In 2007, Guo identified a novel function of GCN2 in regulating lipid metabolism during leucine deprivation. Knockout of the Gcn2 gene in mice results in fatty liver, mediated by high expression of FAS, when the animals are fed a leu- cine-deficient diet for $7 \mathrm{~d}$ [16] and results in insulin resistance following leucine deprivation [27]. Furthermore, Xiao et al. [27] found that GCN2 also plays a critical role in the regulation of insulin sensitivity and glucose metabolism via the mTOR pathway. Taken together, these results strongly suggest that GCN2 is a novel regulator of lipid and glucose metabolic adaptation to deprivation of EAAs. It is a key linker between amino acids, lipids, and glucose metabolism. Further investigations into the mechanisms underlying the activation of GCN2 by leucine deprivation in the liver are required.

\section{$3.2 \quad$ ATF4}

Activating transcription factor 4 (ATF4), also known as CREB2 and C/ATF, belongs to the family of basic zippercontaining proteins $[33,34]$. In mammals, the GCN2/ATF4 pathway has been recognized as the main pathway involved in the regulation of gene expression in response to amino acid deprivation [35-37]. GCN2-dependent phosphorylation of eIF2 $\alpha$ increases in response to the presence of uORF in its $5^{\prime}$ untranslated region (UTR), promoting amino acid biosynthesis and transport upon amino acid deprivation [38]. In addition, accumulating evidence suggests that ATF4 plays an important role in the regulation of the high level of proliferation required during fetal-liver hematopoiesis [39], long-term memory [40], osteoblast differentiation [41], ER stress [42,43], glucose metabolism, and energy homeostasis [44]. With respect to the regulation of glucose metabolism by ATF4, Seo et al. [44] first reported that Atf4-null mice are hypoglycemic, indicating that ATF4 regulates mammalian carbohydrate metabolism and that the Atf4 mutation abrogates diet-induced diabetes as well as hyperlipidemia and hepatosteatosis. All of these Atf4 mutant phenotypes are related to the activation of the mTOR pathway [44]. Another study indicated that ATF4 inhibits insulin secretion and decreases insulin sensitivity through its osteoblastic expression in the liver, fat, and muscle [45]. Recently, Kode et al.

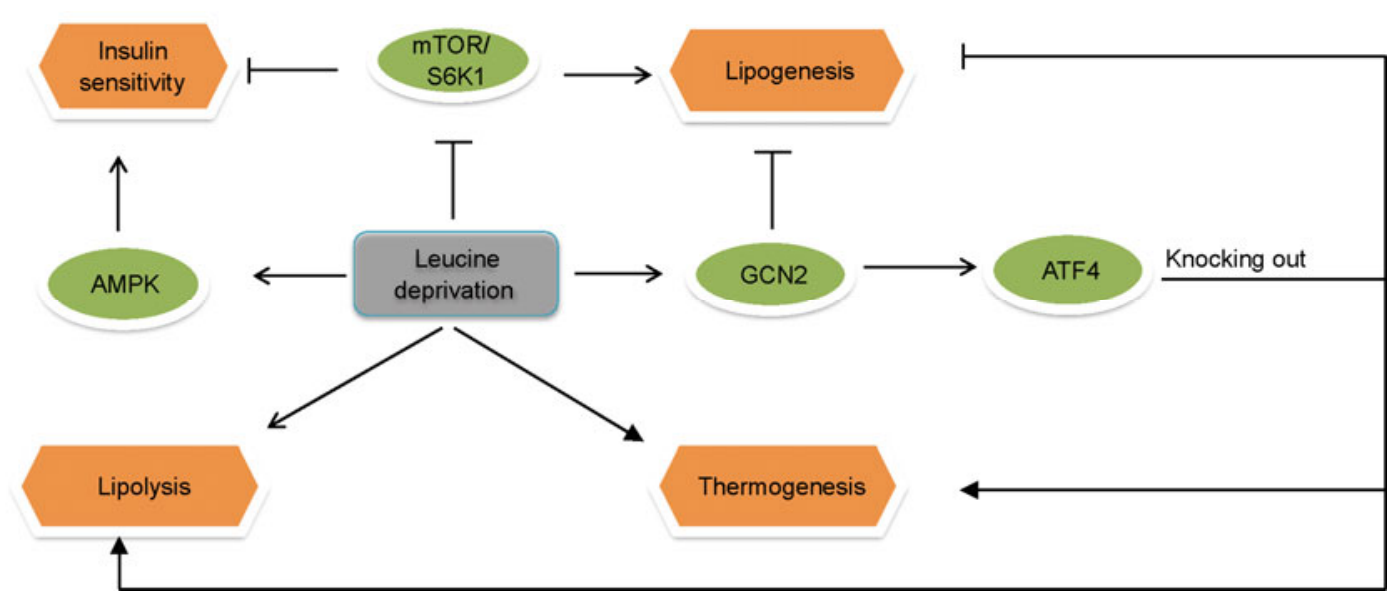

Figure 1 Amino acid sensors and metabolic regulation. 
[46] demonstrated that FoxO1 colocalizes with ATF4 in the osteoblast nucleus and that FOXO1 and ATF4 cooperate in osteoblasts to increase glucose levels and decrease glucose tolerance. All of these results suggested that ATF4 is a novel regulator of glucose metabolism; however, much is still unknown, and the related mechanisms require further investigation.

A recent study in Guo's laboratory has led to the identification of a novel function for ATF4 in regulating lipid metabolism, thermogenesis, and glucose metabolism [47, 48]. Atf4-deficient mice were found to be lean and exhibit increased energy expenditure due to increased thermogenesis in BAT, as described by Seo et al. [44], as well as increased lipolysis and decreased lipogenesis in WAT [47]. When fed on a high-carbohydrate diet (HCD), ATF4deficient mice became resistant to HCD-induced accumulation of triacylglycerols (TAGs) in the liver, and impairment in glucose tolerance via suppression of HCD-induced stearoyl-CoA desaturase 1 (SCD1) expression [48]. Considering of the higher body temperature of ATF4-deficient mice compare to wild-type mice [47], the physiological functions of ATF4 in response to cold stress were also investigated by Wang et al. The results showed that ATF4 is a negative regulator of $\mathrm{PGC} 1 \alpha$ expression through competitive binding with cAMP response element-binding protein (CREB) at a cAMP response element site in the PGC1 $\alpha$ promoter [49]. Because ATF4 is constitutively expressed in a wide variety of tissues, including the brain, heart, WAT, BAT, liver, lungs, and kidneys, we speculated that ATF4 plays a critical role in metabolic regulation. Further investigations are warranted in this regard.

\section{3 mTOR}

mTOR is a serine/threonine protein kinase that has been shown to be essential for cell growth, protein synthesis, cell development, and cell proliferation [17,50,51]. mTOR forms 2 distinct complexes, mTOR complex 1 (mTORC1) and mTOR complex 2 (mTORC2). Studies have shown that mTORC1 is regulated by BCAA availability, especially leucine [52]. Intracerebroventricular injection of leucine can active $\mathrm{mTOR}$ in the hypothalamus, which is involved in the regulation of food intake [17]. In contrast, leucine deprivation suppresses the activity of mTOR in the livers of mice.

Moreover, accumulating evidence has indicated that activation of mTOR/S6 kinase 1 (S6K1) signaling contributes to the development of insulin resistance. The mTOR downstream target S6K1 directly phosphorylates insulin receptor substrate 1 (IRS1) serine residues, including Ser-302/307 [53], Ser-307/312 [54], Ser-632/636 [55], and Ser-1097/Ser1101 [56], which results in the reduced activity of IRS1, thereby impairing PI3K/AKT signaling and increasing insulin resistance [57,58]. As a specific inhibitor of mTORC1, rapamycin can reverse the effects of $\mathrm{mTOR} / \mathrm{S} 6 \mathrm{~K} 1$ activation on insulin resistance [54]. On the other hand, S6K1- null mice exhibit reduced phosphorylation of IRS1 at Ser636 and are resistant to diet-induced insulin resistance [59]. Additional studies have also shown that decreased mTOR/ S6K1 signaling increases insulin sensitivity $[59,60]$. Consistent with these results, our research work had shown that leucine deprivation increases insulin sensitivity via suppressing mTOR activity. Furthermore, overexpression of S6K1 reverses the effects of leucine deprivation on insulin sensitivity in a GCN2-dependent manner [27]. Thus, together with our results, these recent studies have suggested that mTOR acts as a sensor of leucine deficiency downstream of GCN2, thereby regulating insulin sensitivity.

\subsection{AMPK}

AMPK is a crucial cellular energy sensor and signal transducer for maintaining energy homeostasis, a process that is regulated by a wide array of metabolic stresses [61,62]. Recent studies indicate that AMPK also plays a role in the regulation of insulin sensitivity by directly phosphorylating IRS1 [63] or by phosphorylating TSC2, an upstream inhibitor of mTOR [64]. Stimulation of AMPK in skeletal muscle and liver is seen as an exciting prospect for the treatment of type 2 diabetes. AMPK activators, including metformin and rosiglitazone, increase insulin sensitivity and are used clinically in the treatment of type 2 diabetes [65]. Moreover, AMPK has been shown to regulate insulin sensitivity and can be indirectly controlled by nutrient availability $[66,67]$. Indeed, leucine deprivation actives AMPK, contributing to the leucine deprivation-induced increase in insulin sensitivity [27]. In addition, AMPK activity is closely related to amino acid-stimulated insulin secretion [68]. These results indicate that AMPK also acts as a sensor for amino acids. Although it is not known how leucine deprivation activates AMPK, these studies provide new insights into the functions of AMPK in nutritional regulation.

\section{Sensing of amino acids deficiency in the cen- tral nervous system (CNS)}

Amino acid sensing occurs in the gastrointestinal tract, muscle, liver, brain, and other organs. Dysregulation of amino acid sensing induces protein metabolic diseases, metabolic diseases, or cancer. In this review, we focus on the sensing of EAA deficiency in the CNS in the context of metabolic diseases. In the classical behavioral test for EAA deficiency, animals detect and reject a diet lacking an EAA [69]. Indeed, animals detect and reject their first EAAdeficient meal within $20 \mathrm{~min}$ [70]. Lesion studies have shown that an intact anterior piriform cortex (APC) is essential for the detection of EAA deficiency in vivo [71]. Further studies have shown that uncharged tRNA is the primary biochemical sensor of EAA deficiency in the APC and that GCN2 is activated in this process [72]. 
In 2006, Cota et al. [17] found that leucine actives mTOR in the hypothalamus to regulate food intake in rats. It is well established that the hypothalamus plays an important role in regulating energy homeostasis and lipid metabolism [73-75]. Energy homeostasis is maintained by a balance between energy intake and energy expenditure. The hypothalamus has been shown to play a critical role in the regulation of these events by sensing changes in the concentrations of nutrients and hormones and coordinating subsequent physiological responses [20,76,77].

Considering the comprehensive effects of leucine deprivation on energy homeostasis, lipid metabolism, and glucose metabolism, Guo's group investigated the possible involvement of the hypothalamus in regulating these processes. They found that intracerebroventricular administration of leucine significantly attenuated abdominal fat loss via blocking the activation of hormone-sensitive lipase in the WAT and induction of UCP1 in the BAT in leucinedeprived mice. Furthermore, leucine deprivation was found to increase the expression of corticotrophin-releasing hormone $(\mathrm{CRH})$ in the hypothalamus via activation of the stimulatory $\mathrm{G}$ protein/cAMP/protein kinase A/CREB pathway. Finally, the sympathetic nervous system was found to mediate the effect of leucine deprivation on fat loss. These results suggest that hypothalamic $\mathrm{CRH}$ is a critical sensor and transporter for stimulating fat loss upon leucine deprivation [19]. To further elucidate the molecular mechanisms underlying hypothalamic CRH regulation of leucine deprivation-stimulated fat loss, Guo's group used intracerebroventricular injection of adenoviral vectors to identify a novel role for hypothalamic p70 S6K1, a major downstream effector of the leucine-deprivation sensing kinase mTOR, in the stimulation of energy expenditure by leucine deprivation. These studies showed that hypothalamic S6K1 modulates $\mathrm{CRH}$ expression in a melanocortin-4 receptor-dependent manner [78]. Taken together, Guo's studies provide a new perspective for understanding the regulation of energy expenditure by the hypothalamus and the importance of crosstalk between nutritional control and endocrine signal regulation (Figure 2). Whether the hypothalamus senses other amino acids in a manner similar to that for leucine is unknown, and further studies are required to completely elucidate the mechanisms involved.

\section{Conclusion}

In summary, amino acids are novel regulators of nutrition and other metabolic processes and are closely involved in metabolic regulation and the development of metabolic diseases. In these processes, several amino acid sensors, including GCN2, ATF4, mTOR, and AMPK, play pivotal roles in the regulation of lipid metabolism, glucose metabolism, and energy homeostasis. In addition, the hypothalamus, as a core center for metabolic regulation, is also critical for

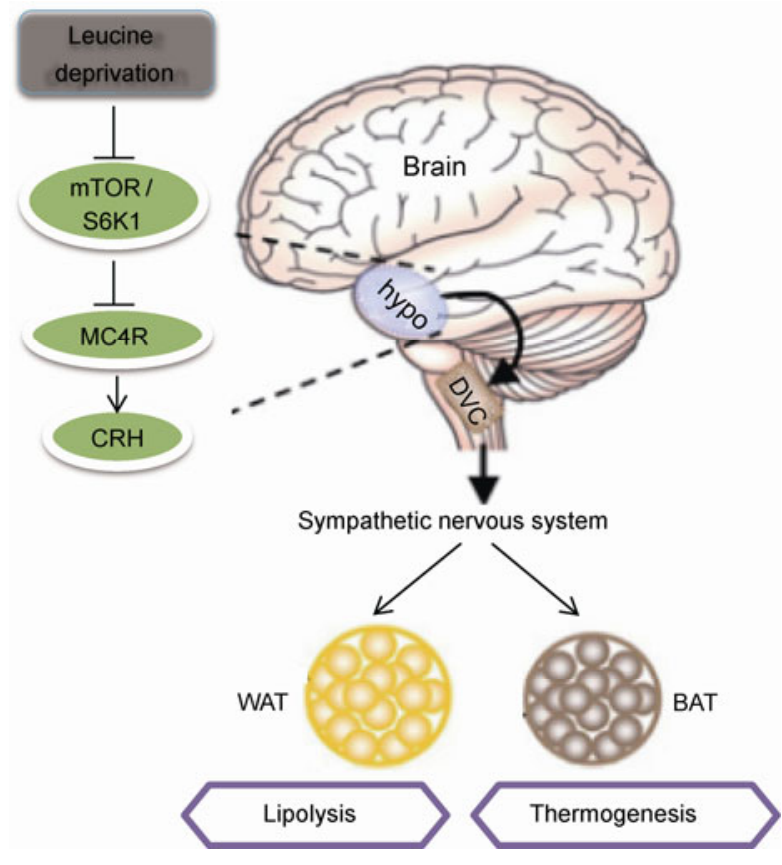

Figure 2 Amino acid sensing in hypothalamus and metabolic regulation.

sensing amino acid levels and mediates the metabolic adaptation of the body upon limitation of EAAs. Although more clinical tests are required to confirm the safety of short- and long-term leucine deprivation and to determine the optimal concentration of leucine in humans, leucine-deficient diets have potential applications in the prevention or treatment of obesity, diabetes, and fatty liver. GCN2, ATF4, mTOR, and AMPK may also be new drug targets for metabolic disease. At present, amino acid sensing in metabolic regulation has become a research hotspot in the metabolic field. From this review, we hope to have presented topics that will be the subject of additional discussion in the future.

1 Wasan K M, Looije N A. Emerging pharmacological approaches to the treatment of obesity. J Pharm Pharm Sci, 2005, 8: 259-271

2 Wild S, Roglic G, Green A, et al. Global prevalence of diabetes: Estimates for the year 2000 and projections for 2030. Diabetes Care, 2004, 27: 1047-1053

3 Jia W P, Wang C, Jiang S, et al. Characteristics of obesity and its related disorders in China. Biomed Environ Sci, 2010, 23: 4-11

4 Yang W, Lu J, Weng J, et al. Prevalence of diabetes among men and women in China. N Engl J Med, 2010, 362: 1090-1101

5 Fan J G, Farrell G C. Epidemiology of non-alcoholic fatty liver disease in China. J Hepatol, 2009, 50: 204-210

6 Hofbauer K G. Molecular pathways to obesity. Int J Obes Relat Metab Disord, 2002, 26 (Suppl 2): S18-27

7 Maejima Y, Iwasaki Y, Yamahara Y, et al. Peripheral oxytocin treatment ameliorates obesity by reducing food intake and visceral fat mass. Aging (Albany NY), 2011, 3: 1169-1177

8 Bruhat A, Jousse C, Fafournoux P. Amino acid limitation regulates gene expression. Proc Nutr Soc, 1999, 58: 625-632

9 Parker B, Noakes M, Luscombe N, et al. Effect of a high-protein, high-monounsaturated fat weight loss diet on glycemic control and lipid levels in type 2 diabetes. Diabetes Care, 2002, 25: 425-430

10 Layman D K, Boileau R A, Erickson D J, et al. A reduced ratio of 
dietary carbohydrate to protein improves body composition and blood lipid profiles during weight loss in adult women. J Nutr, 2003, 133: $411-417$

11 Layman D K, Walker D A. Potential importance of leucine in treatment of obesity and the metabolic syndrome. J Nutr, 2006, 136: 319S-323S

12 Felig P, Marliss E, Cahill G F Jr. Plasma amino acid levels and insulin secretion in obesity. N Engl J Med, 1969, 281: 811-816

13 Newgard C B, An J, Bain J R, et al. A branched-chain amino acidrelated metabolic signature that differentiates obese and lean humans and contributes to insulin resistance. Cell Metab, 2009, 9: 311-326

14 Zhang Y, Guo K, LeBlanc R E, et al. Increasing dietary leucine intake reduces diet-induced obesity and improves glucose and cholesterol metabolism in mice via multimechanisms. Diabetes, 2007, 56: 1647-1654

15 Nairizi A, She P, Vary T C, et al. Leucine supplementation of drinking water does not alter susceptibility to diet-induced obesity in mice. J Nutr, 2009, 139: 715-719

16 Guo F, Cavener D R. The GCN2 eIF2alpha kinase regulates fatty-acid homeostasis in the liver during deprivation of an essential amino acid. Cell Metab, 2007, 5: 103-114

17 Cota D, Proulx K, Smith K A, et al. Hypothalamic mTOR signaling regulates food intake. Science, 2006, 312: 927-930

18 Cheng Y, Meng Q, Wang C, et al. Leucine deprivation decreases fat mass by stimulation of lipolysis in white adipose tissue and upregulation of uncoupling protein 1 (UCP1) in brown adipose tissue. Diabetes, 2010, 59: 17-25

19 Cheng Y, Zhang Q, Meng Q, et al. Leucine deprivation stimulates fat loss via increasing CRH expression in the hypothalamus and activating the sympathetic nervous system. Mol Endocrinol, 2011, 25: $1624-1635$

20 Elmquist J K, Coppari R, Balthasar N, et al. Identifying hypothalamic pathways controlling food intake, body weight, and glucose homeostasis. J Comp Neurol, 2005, 493: 63-71

21 Cypess A M, Lehman S, Williams G, et al. Identification and importance of brown adipose tissue in adult humans. N Engl J Med, 2009, 360: 1509-1517

22 Du Y, Meng Q, Zhang Q, et al. Isoleucine or valine deprivation stimulates fat loss via increasing energy expenditure and regulating lipid metabolism in WAT. Amino Acids, 2012, 43: 725-734

23 She P, Reid T M, Bronson S K, et al. Disruption of BCATm in mice leads to increased energy expenditure associated with the activation of a futile protein turnover cycle. Cell Metab, 2007, 6: 181-194

24 Wang T J, Larson M G, Vasan R S, et al. Metabolite profiles and the risk of developing diabetes. Nat Med, 2011, 17: 448-453

25 Muto Y, Sato S, Watanabe A, et al. Effects of oral branched-chain amino acid granules on event-free survival in patients with liver cirrhosis. Clin Gastroenterol Hepatol, 2005, 3: 705-713

26 Tremblay F, Krebs M, Dombrowski L, et al. Overactivation of S6 kinase 1 as a cause of human insulin resistance during increased amino acid availability. Diabetes, 2005, 54: 2674-2684

27 Xiao F, Huang Z, Li H, et al. Leucine deprivation increases hepatic insulin sensitivity via GCN2/mTOR/S6K1 and AMPK pathways. Diabetes, 2011, 60: 746-756

28 Iwanaka N, Egawa T, Satoubu N, et al. Leucine modulates contractionand insulin-stimulated glucose transport and upstream signaling events in rat skeletal muscle. J Appl Physiol, 2010, 108: 274-282

29 Higuchi N, Kato M, Miyazaki M, et al. Potential role of branchedchain amino acids in glucose metabolism through the accelerated induction of the glucose-sensing apparatus in the liver. J Cell Biochem, 2011, 112: 30-38

30 Meijer A J, Sauerwein H P. Amino acid-dependent signal transduction and insulin sensitivity. Curr Opin Clin Nutr Metab Care, 1999, 2: 207-211

31 Wek S A, Zhu S, Wek R C. The histidyl-tRNA synthetase-related sequence in the eIF-2 alpha protein kinase GCN2 interacts with tRNA and is required for activation in response to starvation for different amino acids. Mol Cell Biol, 1995, 15: 4497-4506

32 Kilberg M S, Pan Y X, Chen H, et al. Nutritional control of gene expression: How mammalian cells respond to amino acid limitation. Annu Rev Nutr, 2005, 25: 59-85

33 Karpinski B A, Morle G D, Huggenvik J, et al. Molecular cloning of human CREB-2: An ATF/CREB transcription factor that can negatively regulate transcription from the cAMP response element. Proc Natl Acad Sci USA, 1992, 89: 4820-4824

34 Vallejo M, Ron D, Miller C P, et al. C/ATF, a member of the activating transcription factor family of DNA-binding proteins, dimerizes with CAAT/enhancer-binding proteins and directs their binding to cAMP response elements. Proc Natl Acad Sci USA, 1993, 90: 4679-4683

35 Carraro V, Maurin A C, Lambert-Langlais S, et al. Amino acid availability controls TRB3 transcription in liver through the GCN2/eIF2alpha/ATF4 pathway. PLoS One, 2010, 5: e15716

36 Kilberg M S, Shan J, Su N. ATF4-dependent transcription mediates signaling of amino acid limitation. Trends Endocrinol Metab, 2009, 20: $436-443$

37 Averous J, Bruhat A, Jousse C, et al. Induction of CHOP expression by amino acid limitation requires both ATF4 expression and ATF2 phosphorylation. J Biol Chem, 2004, 279: 5288-5297

38 Harding H P, Novoa I, Zhang Y, et al. Regulated translation initiation controls stress-induced gene expression in mammalian cells. Mol Cell, 2000, 6: 1099-1108

39 Masuoka H C, Townes T M. Targeted disruption of the activating transcription factor 4 gene results in severe fetal anemia in mice. Blood, 2002, 99: 736-745

40 Mohamed H A, Yao W, Fioravante D, et al. cAMP-response elements in Aplysia creb1, creb2, and Ap-uch promoters: Implications for feedback loops modulating long term memory. J Biol Chem, 2005, 280: 27035-27043

41 Yang X, Matsuda K, Bialek P, et al. ATF4 is a substrate of RSK2 and an essential regulator of osteoblast biology; implication for CoffinLowry Syndrome. Cell, 2004, 117: 387-398

42 Cao J, Dai D L, Yao L, et al. Saturated fatty acid induction of endoplasmic reticulum stress and apoptosis in human liver cells via the PERK/ATF4/CHOP signaling pathway. Mol Cell Biochem, 2012, 364: $115-129$

43 Ohoka N, Yoshii S, Hattori T, et al. TRB3, a novel ER stressinducible gene, is induced via ATF4-CHOP pathway and is involved in cell death. EMBO J, 2005, 24: 1243-1255

44 Seo J, Fortuno E S, 3rd, Suh J M, et al. Atf4 regulates obesity, glucose homeostasis, and energy expenditure. Diabetes, 2009, 58: 2565-2573

45 Yoshizawa T, Hinoi E, Jung D Y, et al. The transcription factor ATF4 regulates glucose metabolism in mice through its expression in osteoblasts. J Clin Invest, 2009, 119: 2807-2817

46 Kode A, Mosialou I, Silva B C, et al. FoxO1 protein cooperates with ATF4 protein in osteoblasts to control glucose homeostasis. J Biol Chem, 2012, 287: 8757-8768

47 Wang C, Huang Z, Du Y, et al. ATF4 regulates lipid metabolism and thermogenesis. Cell Res, 2010, 20: 174-184

48 Li H, Meng Q, Xiao F, et al. ATF4 deficiency protects mice from high-carbohydrate-diet-induced liver steatosis. Biochem J, 2011, 438: 283-289

49 Wang C, Xia T, Du Y, et al. Effects of ATF4 on PGC1 $\alpha$ expression in brown adipose tissue and metabolic responses to cold stress. Metabolism, 2012, doi:10.1016/j.metabol.2012.07.017

50 Wullschleger S, Loewith R, Hall M N. TOR signaling in growth and metabolism. Cell, 2006, 124: 471-484

51 Inoki K, Corradetti M N, Guan K L. Dysregulation of the TSCmTOR pathway in human disease. Nat Genet, 2005, 37: 19-24

52 Lynch C J. Role of leucine in the regulation of mTOR by amino acids: Revelations from structure-activity studies. J Nutr, 2001, 131: $861 \mathrm{~S}-865 \mathrm{~S}$

53 Harrington L S, Findlay G M, Gray A, et al. The TSC1-2 tumor suppressor controls insulin-PI3K signaling via regulation of IRS proteins. J Cell Biol, 2004, 166: 213-223

54 Carlson C J, White M F, Rondinone C M. Mammalian target of rapamycin regulates IRS-1 serine 307 phosphorylation. Biochem Biophys Res Commun, 2004, 316: 533-539 
55 Ozes O N, Akca H, Mayo L D, et al. A phosphatidylinositol 3-kinase/ Akt/mTOR pathway mediates and PTEN antagonizes tumor necrosis factor inhibition of insulin signaling through insulin receptor substrate-1. Proc Natl Acad Sci USA, 2001, 98: 4640-4645

56 Tremblay F, Brule S, Hee Um S, et al. Identification of IRS-1 Ser-1101 as a target of S6K1 in nutrient- and obesity-induced insulin resistance. Proc Natl Acad Sci USA, 2007, 104: 14056-14061

57 Draznin B. Molecular mechanisms of insulin resistance: Serine phosphorylation of insulin receptor substrate-1 and increased expression of p85alpha: The two sides of a coin. Diabetes, 2006, 55: 2392-2397

58 Pederson T M, Kramer D L, Rondinone C M. Serine/threonine phosphorylation of IRS-1 triggers its degradation: Possible regulation by tyrosine phosphorylation. Diabetes, 2001, 50: 24-31

59 Um S H, Frigerio F, Watanabe M, et al. Absence of S6K1 protects against age- and diet-induced obesity while enhancing insulin sensitivity. Nature, 2004, 431: 200-205

60 Krebs M, Brunmair B, Brehm A, et al. The Mammalian target of rapamycin pathway regulates nutrient-sensitive glucose uptake in man. Diabetes, 2007, 56: 1600-1607

61 Hardie D G, Ross F A, Hawley S A. AMPK: A nutrient and energy sensor that maintains energy homeostasis. Nat Rev Mol Cell Biol, 2012, 13: 251-262

62 Hardie D G, Sakamoto K. AMPK: A key sensor of fuel and energy status in skeletal muscle. Physiology (Bethesda), 2006, 21: 48-60

63 Jakobsen S N, Hardie D G, Morrice N, et al. 5'-AMP-activated protein kinase phosphorylates IRS-1 on Ser-789 in mouse C2C12 myotubes in response to 5-aminoimidazole-4-carboxamide riboside. J Biol Chem, 2001, 276: 46912-46916

64 Inoki K, Zhu T, Guan K L. TSC2 mediates cellular energy response to control cell growth and survival. Cell, 2003, 115: 577-590

65 Zhang B B, Zhou G, Li C. AMPK: An emerging drug target for diabetes and the metabolic syndrome. Cell Metab, 2009, 9: 407-416

66 Ruderman N, Prentki M. AMP kinase and malonyl-CoA: Targets for therapy of the metabolic syndrome. Nat Rev Drug Discov, 2004, 3:
340-351

67 Long Y C, Zierath J R. AMP-activated protein kinase signaling in metabolic regulation. J Clin Invest, 2006, 116: 1776-1783

68 Leclerc I, Rutter G A. AMP-activated protein kinase: A new beta-cell glucose sensor? Regulation by amino acids and calcium ions. Diabetes, 2004, 53 (Suppl 3): S67-74

69 Harper A E, Benevenga N J, Wohlhueter R M. Effects of ingestion of disproportionate amounts of amino acids. Physiol Rev, 1970, 50: 428-558

70 Gietzen D W, Hao S, Anthony T G. Mechanisms of food intake repression in indispensable amino acid deficiency. Annu Rev Nutr, 2007, 27: 63-78

71 Leung P M, Rogers Q R. Importance of prepyriform cortex in foodintake response of rats to amino acids. Am J Physiol, 1971, 221: 929-935

72 Hao S, Ross-Inta C M, Gietzen D W. The sensing of essential amino acid deficiency in the anterior piriform cortex, that requires the uncharged tRNA/GCN2 pathway, is sensitive to wortmannin but not rapamycin. Pharmacol Biochem Behav, 2010, 94: 333-340

73 Williams G, Bing C, Cai X J, et al. The hypothalamus and the control of energy homeostasis: Different circuits, different purposes. Physiol Behav, 2001, 74: 683-701

74 Tomaszuk A, Simpson C, Williams G. Neuropeptide Y, the hypothalamus and the regulation of energy homeostasis. Horm Res, 1996, 46: 53-58

75 Shimazu T. The hypothalamus and metabolic regulation, especially hypothalamic regulation of lipid metabolism and its disorder. Tanpakushitsu Kakusan Koso, 1984, 29: 1589-1599

76 Blouet C, Schwartz G J. Hypothalamic nutrient sensing in the control of energy homeostasis. Behav Brain Res, 2010, 209: 1-12

77 Cota D, Proulx K, Seeley R J. The role of CNS fuel sensing in energy and glucose regulation. Gastroenterology, 2007, 132: 2158-2168

78 Xia T, Cheng Y, Zhang Q, et al. S6K1 in the central nervous system regulates energy expenditure via MC4R/CRH pathways in response to deprivation of an essential amino acid. Diabetes, 2012, 61: 24612471

Open Access This article is distributed under the terms of the Creative Commons Attribution License which permits any use, distribution, and reproduction in any medium, provided the original author(s) and source are credited. 